\title{
Oxigenación con membrana extracorpórea y COVID-19
}

\section{Extracorporeal membrane Oxygenation and COVID-19}

Rafael Lima Linares ${ }^{1}$

\begin{abstract}
ECMO (ExtraCorporeal Membrane Oxygenation) is still a "rescue" therapy for both hemodynamic and respiratory support. Its use is relatively low and is restricted to specialized centers. The WHO declared as pandemic the severe acute respiratory syndrome due to coronavirus 2 (SARS-CoV-2) causing fatal pneumonia due to coronavirus 19 disease (COVID-19). Although the new coronavirus has a low lethality compared to other epidemics, its diffusion capacity is phenomenal. When ECMO therapy is indicated, its initiation in a specialized ECMO center should not be delayed. HEALTH WORKERS ARE OF HIGH PRIORITY. VA or VAV support may be indicated. In Mexico, we have even considered activating the logistics of obtaining an ECMO team since the patient presents with $\mathrm{PaO}_{2} / \mathrm{FiO}_{2}$ of $100 \mathrm{mmHg}$ or when it is indicated to pronate the patient. The early use and early activation of an ECMO team will undoubtedly give better results than the late application of this therapy. Direct myocardial involvement due to this virus has been reported causing myocarditis and acute coronary events, so it may be worth leaving a femoral artery vascular introducer so that if necessary, the patient can be supported with VA ECMO or VAV ECMO. These measures may lead to improved prognosis and outcome for patients affected by COVID-19.
\end{abstract}

\section{RESUMEN}

ECMO (ExtraCorporeal Membrane Oxygenation) es una terapia de "rescate" para soporte, tanto hemodinámico como respiratorio. Su uso es relativamente bajo y está restringido a centros especializados. La OMS declaró como pandémico el síndrome agudo respiratorio severo por coronavirus 2 (SARS-CoV-2) causante de neumonía fatal por la enfermedad del coronavirus 19 (COVID-19).
Key words:

ECMO, COVID-19

Palabras clave:

ECMO, COVID-19

Anestesiólogo Cardiovascular. Instituto Nacional de Cardiología "Ignacio Chávez", UMAE Cardiología.

Centro Médico Nacional Siglo XXI.

Fecha de recepción: 24 de marzo de 2020

Fecha de aceptación: 26 de marzo de 2020

\section{ORCID}

https://orcid.org/0000-0001-5391-2626

Correspondencia:

Rafael Lima Linares

Email: drrafaellima@hotmail.com 
Aunque el nuevo coronavirus presenta una baja letalidad en comparación con otras epidemias, su capacidad de difusión es fenomenal. Cuando la terapia con ECMO está indicada, no debe retrasarse su inicio en un centro ECMO especializado. LOS TRABAJADORES DE LA SALUD SON DE ALTA PRIORIDAD. El soporte VA o VAV puede indicarse. En México hemos considerado incluso activar la logística de conseguir en ECMO team desde encontrarnos con $\mathrm{PaO}_{2} /$ $\mathrm{FiO}_{2}$ de $100 \mathrm{mmHg}$ o cuando se indica pronar al paciente. El uso temprano y la activación temprana de un ECMO team, sin duda dará mejores resultados que la aplicación tardía de esta terapia. Se ha reportado afección miocárdica directa por este virus provocando miocarditis y eventos coronarios agudos, por lo que podría valer la pena dejar un introductor vascular arterial femoral para que en caso de ser necesario se pueda soportar al paciente con ECMO VA o ECMO VAV. Estas medidas pueden llevar a mejorar el pronóstico y desenlace de los pacientes afectados por COVID-19.

\section{E} I término ECMO (ExtraCorporeal Membrane Oxygenation) se usa habitualmente para referirse a diferentes soportes cardíaco, pulmonar o ambos. ECMO es una terapia de "rescate" para soporte, tanto hemodinámico como respiratorio, actualmente es desconocido el número de pacientes que desarroIlarán un SDRA tan severo que requerirán este tipo de soporte. Su uso es relativamente bajo y está restringido a centros especializados[1],[4].

Este soporte se desarrolló a partir de la máquina de circulación extracorpórea usada por primera vez por Gibbon en 1953 en la clínica Mayo, fue hasta 1971 cuando se usó por primera vez como soporte pulmonar fuera de la sala de quirófano[5] después, de 1975 a 1985, se perfeccionó la circulación extracorpórea para falla respiratoria neonatal, en esta población su uso creció rápidamente por que tras unos días de asistencia, las condiciones fisiológicas del paciente permitían que el desenlace pasara de $90 \%$ de mortalidad a $90 \%$ de sobrevida saludable[6]. Después se extendió su uso a la población adulta tanto para soporte respiratorio como circulatorio en 1990 la indicación principal era en soporte respiratorio en pacientes neonatales, pero hacia 2018 la mayoría de los casos eran reportados como soporte circulatorio en los registros de la ELSO (Extracorporeal Life Support Organization). En 2009 se realizó el estudio CESAR[7] $y$, posteriormente, el EOLIA[8] fallando en demostrar estadísticamente que ECMO en SDRA reducía la mortalidad, pero sí demostraron que esta terapia no provocaba mayor daño, EOLIA, además, demostró que el ECMO puede ser beneficioso no solo como terapia de rescate, sino que su uso en etapas tempranas del SDRA puede ser beneficioso[4].
La OMS declaró como pandémico el síndrome agudo respiratorio severo por coronavirus 2 (SARSCoV-2) causante de neumonía fatal por la enfermedad del coronavirus 19 (COVID-19)[1]. Esta nueva enfermedad que se inició en China desde 2019 y que para finales de febrero de 2020 había alcanzado más de 68.000 casos con más de 1.600 fatalidades[9] recibiendo reportes de pacientes que necesitaron soporte con una membrana de oxigenación extracorpórea (ECMO). Esta pandemia está sucediendo en un momento en el que la infraestructura y los recursos en educación e investigación están considerablemente más evolucionados y organizados que hace una década y más importante aún, hay sustancialmente más evidencia que apoya la eficacia y seguridad del soporte con ECMO en pacientes con SDRA[1].

Aunque el nuevo coronavirus presenta una baja letalidad en comparación con otras epidemias, su capacidad de difusión es fenomenal, cabe hacer notar que el screening con base en síntomas y signos es inefectivo, ya que personas asintomáticas pueden transmitir el virus[2]. En las etapas iniciales de la enfermedad se publicó un llamado a la acción en el Lancet Respiratory Medecine[2] con una recomendación especial para la preparación de equipos especializados en soporte extracorpóreo[2].

Este ECMO team debe conformarse por personal entrenado específicamente en el cuidado de pacientes con soporte con ECMO, debe establecerse una "cadena de mando" a nivel central y regional estableciendo funciones específicas para cada miembro del equipo. Se debe entrenar a este personal en la correcta utilización del equipo de protección personal y el manejo de secreciones de los pacientes, actualizar 
la disponibilidad de máquinas y consumibles, todos los centros ECMO deben tener un lugar designado para la colocación y remoción del equipo de protección personal, respiradores purificadores de aire y debe existir un plan para cada centro con capacidad de atender pacientes con ECMO donde se establezcan procedimientos estrictos de control de infecciones, transporte de pacientes y apropiado desecho de basura, planes de comunicación, referencia, contrarreferencia y reporte de pacientes, así como planes de contingencia, el destete y rehabilitación de pacientes que tuvieron soporte con ECMO deben realizarse con estrictos controles de infección y protección personal, así mismo el cuidado y la decanulación post mortem. Las consideraciones éticas incluyen la racionalización apropiada del uso de ECMO en lo que corresponde a sus indicaciones, pero también cuándo continuar el soporte o terminarlo, en casos desafiantes será necesario incluso el apoyo del Departamento de Ética y Médico Legal del hospital. Finalmente, es necesario mantener la calidad de la atención, recabar y compartir los datos y experiencia generados en el cuidado de estos pacientes[2].

Recientemente y con base en la evidencia internacional, ELSO publicó su documento guía para el manejo con ECMO de pacientes con COVID-19 con falla cardiorrespiratoria severa haciendo hincapié en que, cuando la terapia con ECMO está indicada, no debe retrasarse su inicio en un centro ECMO especializado, tomando en cuenta diferentes consideraciones:

1. ECMO debe considerarse para los pacientes con COVID-19. Ésta será una decisión local que deberá tomarse caso a caso y reevaluarse regularmente con base en la carga de trabajo, staff del hospital, otros recursos, apoyo gubernamental y políticas intrahospitalarias. Si el hospital debe orientar sus recursos para otros pacientes, la terapia con ECMO no deberá considerarse sino hasta que los recursos hospitalarios se estabilicen. Si el hospital siente que puede ofrecer esta terapia de forma segura deberá ofrecerse a pacientes con buen pronóstico con ECMO, la indicación en pacientes con edad avanzada, múltiples comorbilidades y falla orgánica múltiple, debería ser rara.

2. ECPR. ECMO en paro cardiorrespiratorio implica un entrenamiento complejo y extenso, los centros que no proveen este servicio no deberán iniciarlo. En centros experimentados podrá ofrecerse este servicio siempre considerando la escasez potencial de recursos y la potencial contaminación del personal involucrado. En pacientes con múltiples comorbilidades, la indicación de ECPR debería ser rara.
3. ECMO para indicaciones habituales. Su uso deberá restringirse para cuando los recursos hospitalarios los cubran.

4. Quiénes son prioridad: pacientes con pocas o nulas comorbilidades. LOS TRABAJADORES DE LA SALUD SON DE ALTA PRIORIDAD. Conforme los recursos cambien las prioridades de atención deberán cambiar.

5. Quiénes deberán excluirse. Las exclusiones estándar aplican, tal como la enfermedad terminal, daño al sistema nervioso central, indicación de no resucitar, rechazo de esta terapia. La exclusión de pacientes con COVID-19 será una decisión hospitalaria local, pacientes con comorbilidades deberán excluirse, en pacientes con edad avanzada deberá balancearse contra la disponibilidad de recursos, los pacientes con ventilación mecánica por más de 7 días deberán excluirse,

6. Futilidad. No todos los pacientes en ECMO van a mejorar, la evaluación clínica deberá revalorarse continuamente, en momentos de recursos limitados si no hay recuperación en 21 días aproximadamente deberá discontinuarse el tratamiento y regresar a manejo convencional.

7. Falla cardíaca. La hipotensión sostenida a pesar de otro manejo médico debe evaluarse por medio de signos clínicos y ecocardiográficos, el soporte VA o VAV puede indicarse, por lo que la evaluación ecocardiográfica deberá repetirse cada tanto en caso de sospecha clínica de disfunción cardía$\mathrm{ca}[10]$.

Como en la aplicación de todas las guías, siempre se debe considerar la situación local y regional donde el soporte con ECMO es necesario, en algunos hospitales en México, dada la poca disponibilidad de centros ECMO con experiencia o de dispositivos en la mayoría de los hospitales, hemos considerado incluso activar la logística de conseguir en ECMO team desde encontrarnos con $\mathrm{PaO}_{2} / \mathrm{FiO}_{2}$ de $100 \mathrm{mmHg} \mathrm{O}$ apenas menos de $100 \mathrm{mmHg}$ o cuando se indica pronar al paciente, esto no es una indicación absoluta de soportar al paciente, pero sí de tener en aviso al equipo correspondiente. El tener disponible un dispositivo de ECMO en cada hospital y personal entrenado en su uso aún no es una realidad, sin embargo, el uso temprano y la activación temprana de un ECMO team, sin duda dará mejores resultados que la aplicación tardía de esta terapia.

Específicamente en pacientes con COVID-19, el abordaje al igual que en otros pacientes con algún tipo de SDRA severo deberá hacerse con ECMO VV (Cuadro 1), cuando se haya verificado por paráme- 


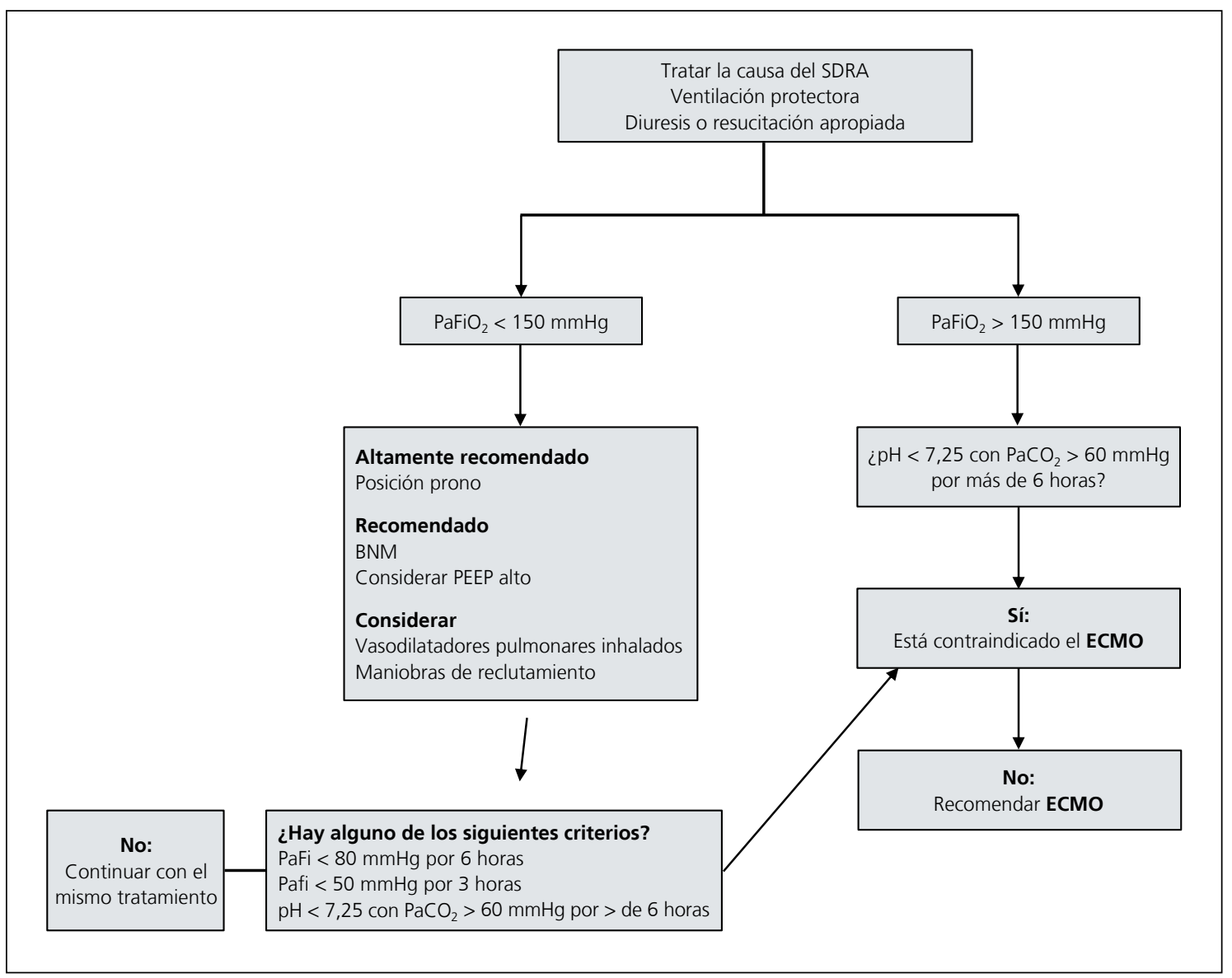

Cuadro 1. Manejo del SDRA.

tros clínicos y ecocardiográficos que la función cardíaca está conservada o requiere dosis bajas de fármacos vaso o cardioactivos, sin embargo, a diferencia de otros microorganismos, se ha reportado afección miocárdica directa por este virus provocando miocarditis y eventos coronarios agudos, por lo que podría valer la pena dejar un introductor vascular arterial femoral para que en caso de ser necesario se pueda soportar al paciente con ECMO VA o ECMO VAV sin realizar punciones nuevas, es mandatorio el conoci- miento (al menos básico) de ecocardiografía y ultrasonido vascular para asegurar los accesos vasculares y valorar la función cardíaca cada tanto por cualquier personal en contacto con este tipo de pacientes. Sin duda, muchas cosas van a cambiar conforme el avance de esta terrible enfermedad, pero por el momento con base en la información disponible actual estas medidas pueden llevar a mejorar el pronóstico y desenlace de los pacientes afectados por COVID-19. 


\section{Referencias}

1. Ramanathan $K$, Antognini $D$, Combes A, Paden M, Zakhary $B$, Ogino $M$, et al. Planning and provision of ECMO services for severe ARDS during the COVID-19 pandemic and other outbreaks of emerging infectious disease. Lancet Respir Med. 2020 Mar. https:// doi.org/10.1016/S22132600(20)30121-1.

2. Ronco C, Reis T, De Rosa S. Coronavirus Epidemic and Extracorporeal Therapies in Intensive Care: si vis pacem para bellum. Blood Purif. 2020 Mar;1-4. https://doi. org/10.1159/000507039 PMID:32172242

3. Ronco C, Navalesi P, Vincent JL. Coronavirus epidemic: preparing for extracorporeal organ support in intensive care. Lancet Respir Med. 2020 Mar;8(3):240-1. https://doi.org/10.1016/ S2213-2600(20)30060-6 PMID:32035509

4. Quintel M, Bartlett RH, Grocott MPW, Combes A, Ranieri MV, Baiocchi M, et al. Extracorporeal Membrane Oxygenation for Respiratory Failure. Anesthesiology. Ovid Technologies (Wolters Kluwer Health); 2020 May;132(5):125776. http://dx.doi.org/10.1097/ aln.0000000000003221.

5. Hill JD, O'Brien TG, Murray JJ, Dontigny L, Bramson ML, Osborn JJ, et al. Prolonged extracorporeal oxygenation for acute post-traumatic respiratory failure (shock-lung syndrome). Use of the Bramson membrane lung. N Engl J Med. 1972 Mar;286(12):629-34. https://doi.org/10.1056/ NEJM197203232861204 PMID:5060491

6. Bartlett RH. Esperanza: the first neonatal ECMO patient. ASAIO J. 2017 Nov/Dec;63(6):832-43. https://doi.org/10.1097/ MAT.0000000000000697 PMID:29084039

7. Peek GJ, Mugford M, Tiruvoipati R, Wilson A, Allen E, Thalanany MM, et al.; CESAR trial collaboration. Efficacy and economic assessment of conventional ventilatory support versus extracorporeal membrane oxygenation for severe adult respiratory failure (CESAR): a multicentre randomised controlled trial. Lancet. 2009 Oct;374(9698):1351-63. https://doi.org/10.1016/ S0140-6736(09)61069-2 PMID:19762075

8. Combes A, Hajage D, Capellier G, Demoule A, Lavoué S, Guervilly $C$, et al.; EOLIA Trial Group, REVA, and ECMONet. REVA, and ECMONet: extracorporeal membrane oxygenation for severe acute respiratory distress syndrome. N Engl J Med. 2018 May;378(21):1965-75. https://doi.org/10.1056/ NEJMoa1800385 PMID:29791822

9. Li Min. Extracorporeal membrane oxygenation support in 2019 novel coronavirus disease: indications, timing, and implementation. Chinese Medical Journal, Publish Ahead of Print

10. ELSO Guidance Document: ECMO for COVID-19 Patients with Severe Cardiopulmonary Failure 